Editorial

\title{
Tranexamic acid - a recipe for saving lives in traumatic bleeding
}

Professor Ian Roberts ${ }^{1,2}$

Using kitchen scales, carefully weight out $4 \mathrm{~kg}$ of rice and pour it into a deep saucepan. Now put your hands into the rice and let the grains run between your fingers. Contemplate carefully each grain. The number of grains (about 140 000) is approximately the number of lives that could be saved each year worldwide if all hospitalized trauma patients with significant bleeding were treated with tranexamic acid (TXA) within 3 hours of injury. TXA is cheap and widely available. All that is needed to reap these human benefits is that doctors use it.

That TXA is a potent inhibitor offibrinolysis was first reported by Shosuke and Utako Okamoto in the Keio Journal of Medicine in September 1962 [1]. Since then, TXA has been widely used to treat heavy menstrual bleeding and to reduce blood loss in elective surgery where it reduces blood transfusion by about one third [2]. The CRASH-2 collaborators hypothesized that TXA might also reduce bleeding in trauma patients. The CRASH-2 trial was a UK government funded randomized trial of the effects of the early administration of TXA on death, vascular occlusive events and blood transfusion in bleeding trauma patients.

A total of 20211 adults with significant traumatic bleeding were randomized to receive TXA or matching placebo, with 99.6\% follow-up. The risk of death due to bleeding was significantly reduced with TXA. If TXA is given within 3 hours of injury, it reduces the risk of bleeding to death by nearly one-third (relative risk $=0.72[95 \% \mathrm{CI} 0.63-0.83], P<0.001$ ). All cause mortality was also significantly reduced [3,4]. The large numbers of patients studied in a wide range of different health care settings help these results to be generalized widely. On the basis of the results of the CRASH-2 trial, TXA has been included in the World Health Organization (WHO) list of essential medicines [5]. Giving TXA to bleeding trauma patients within 3 hours of the injury could save over 100000 lives per year worldwide. Giving TXA to bleeding trauma patients is highly cost-effective in high, middle and low income countries [6]. It is essential that all doctors who treat trauma patients are aware of the results of the CRASH-2 trial.

TXA should be given to all adults with significant haemorrhage ( $\mathrm{SBP}<90, \mathrm{HR}>110$ ) or those considered by the clinician to be at risk for significant haemorrhage. Because the effect of TXA on death due to bleeding depends importantly on the time interval between the injury and the onset of treatment, it should be given as early as possible and within 3 or 4 hours of the injury as it is unlikely to be effective if given later than this.

\section{References}

1. Okamoto S, Okamoto U. Amino-methyl-cyclohexane-carbolic acid: AMCHA. A new potent inhibitor of fibrinolysis. Keio Journal of Medicine, 1962, 11:105-115.

2. Henry DA et al. Antifibrinolytic use for minimising perioperative allogeneic blood transfusion. Cochrane Database of Systematic Reviews, 2007, 4: CD001886.

3. The CRASH-2 Collaborators. Effects of tranexamic acid on death, vascular occlusive events, and blood transfusion in trauma patients with significant haemorrhage (CRASH-2): a randomized, placebo-controlled trial. Lancet, 2010, 376:23-32.

4. The CRASH-2 Collaborators. The importance of early treatment with tranexamic acid in bleeding trauma patients: an exploratory analysis of the CRASH-2 randomized controlled trial. Lancet, 2011, 377:1096-1101.

5. Summary of the report of the 18th meeting of the WHO Expert Committee on the Selection and Use of Essential Medicines (http://www.who.int/selection_medicines/committees/ expert/18/en/index.html, accessed 22 August 2011).

6. Guerriero $\mathrm{C}$ et al. Cost-effectiveness analysis of administering tranexamic acid to bleeding trauma patients using evidence from the CRASH-2 Trial. PLoS ONE, 2011, 6(5):e18987. 
